\title{
Occulting Ozone Observatory Science Overview
}

\author{
Dmitry Savransky ${ }^{a}$, David N. Spergel ${ }^{a}$, N. Jeremy Kasdin ${ }^{a}$, Eric J. Cady $^{a}$, \\ P. Douglas Lisman ${ }^{b}$, Steven H. Pravdo ${ }^{b}$, Stuart B. Shaklan ${ }^{b}$, Yuka Fujii ${ }^{c}$ \\ ${ }^{a}$ Princeton University, Princeton, NJ USA \\ ${ }^{b}$ Jet Propulsion Lab and California Institute of Technology, Pasadena, CA USA \\ ${ }^{c}$ The University of Tokyo, Tokyo, Japan
}

\begin{abstract}
We present an analysis of the Occulting Ozone Observatory $\left(\mathrm{O}_{3}\right)$ - a $\$ 1$ billion class mission dedicated to finding extra-solar planets down to Earth size, performing photometric characterizations of planets and disks, detecting the presence of ozone, and general astrophysics. We present trade studies for the observatory, composed of a 1 to $2 \mathrm{~m}$ telescope based on heritage imaging systems and a complementary sized, free-flying occulter spacecraft, to maximize the expected science yield for this mission class. Using a camera with four filters each in the 250$550 \mathrm{~nm}$ and 500-1100 nm bands, this modest-size telescope can detect atmospheric ozone in Earth-like planets, methane in gas giants, determine planetary spin rotation periods, characterize the surface composition of rocky planets and determine or constrain the values of basic orbital elements. We present multiple different mission designs along with the expected number of planetary detections and photometric characterizations.
\end{abstract}

Keywords: exoplanets, mission simulation, occulters

\section{INTRODUCTION}

The last decade's successes in ground-based exoplanet finding, ${ }^{1}$ the first results from operating space-based planet finders such as Kepler, ${ }^{2}$ and the first-ever images of exoplanets ${ }^{3,4}$ have all made a dedicated, space-based exoplanet imaging instrument a key goal for a large part of the exoplanetary science community. Over the last few years, legacy designs such as TPF-C ${ }^{5}$ and TPF-I ${ }^{6}$ have been joined by new concepts and technologies, such as free-flying occulter based missions including THEIA ${ }^{7}$ and the New Worlds Observer. ${ }^{8}$ Much time and effort has been expended not only on generating and improving mission concepts, but also on meticulously modeling them and incorporating as much new information about known exosystems as possible in order to ensure the success of these future missions. Unfortunately, developing and flying any one of these mission concepts would represent an enormous investment of time and funds, and the reality of limited resources is such that it would be unlikely for more than one such mission to be scheduled for the coming decade.

Could a smaller mission achieve many of the science goals of a flagship mission? Can we detect nearby "Earths" by building on existing hardware and using existing launch vehicles? The appeal of taking this approach is two-fold: First, building upon legacy hardware is always preferable to starting from scratch, provided that the existing hardware is capable of doing the intended task. Second, if it can be demonstrated that a design can provide a portion of the science expected from a flagship-class mission, for one fifth or one quarter of the price, there is a higher likelihood that one or more such designs will be funded. Flying multiple, different small-scale missions may actually be preferable to one flagship-class mission as it would advance the technology readiness levels of many key technologies, and would allow the community to settle long standing debates as to the efficacy of competing technology paths (like, for example, internal coronagraphs versus external occulters).

The task remains, then, to create a scaled down planet-finder, and to demonstrate that it has a high probability of providing us with useful scientific data. This paper will explore the capabilities of one such concept - the Occulting Ozone Observatory (henceforth $\mathrm{O}_{3}$ ). The observatory, composed of a 1 to $2 \mathrm{~m}$ telescope based on existing and proven systems, and a free-flying occulter, is expected to cover the same wavelength range and be capable of detecting the same classes of planets as the $4 \mathrm{~m}$ THEIA, or $8 \mathrm{~m}$ TPF-C. Of course, the smaller aperture increases required integration times and makes high resolution spectroscopy very difficult, which led to

Send correspondence to Dmitry Savransky dsavrans@princeton.edu

Space Telescopes and Instrumentation 2010: Optical, Infrared, and Millimeter Wave, edited by Jacobus M. Oschmann Jr., Mark C. Clampin, Howard A. MacEwen, Proc. of SPIE Vol. $7731,77312 \mathrm{H} \cdot$ (C) 2010 SPIE · CCC code: 0277-786X/10/\$18 - doi: 10.1117/12.857375

Proc. of SPIE Vol. $773177312 \mathrm{H}-1$ 
a decision to forgo spectroscopic capability in favor of photometric characterization only. The current $\mathrm{O}_{3}$ design is presented in $\S 2$, its overall science capabilities are explored in $\S 3$, and ongoing work in mission simulation and analysis is detailed in $\S 4$.

\section{OBSERVATORY DESIGN}

When first presented, ${ }^{9}$ the $\mathrm{O}_{3}$ system was composed of a $1.1 \mathrm{~m}$ telescope (modeled after the existing ITTNextView Program satellites such as GeoEye-1) and a $30 \mathrm{~m}$ diameter occulter. Subsequent analysis, however, indicated that the tolerances on occulter manufacturing requirements imposed by the relatively small telescope aperture were very tight, prompting us to study larger telescopes up to $2 \mathrm{~m}$ (which will be the aperture size used for the studies described in $\S 4$ ). The occulter was also redesigned to accommodate a larger telescope, resulting in a diameter of $34 \mathrm{~m}$. As an additional way of easing manufacturing and positioning requirements, the geometric inner working angle (IWA) was relaxed from 75 mas to 90 mas, thereby reducing the separation distance between occulter and telescope. For a detailed discussion on tolerancing and error budgeting for occulters, see Ref. 10.
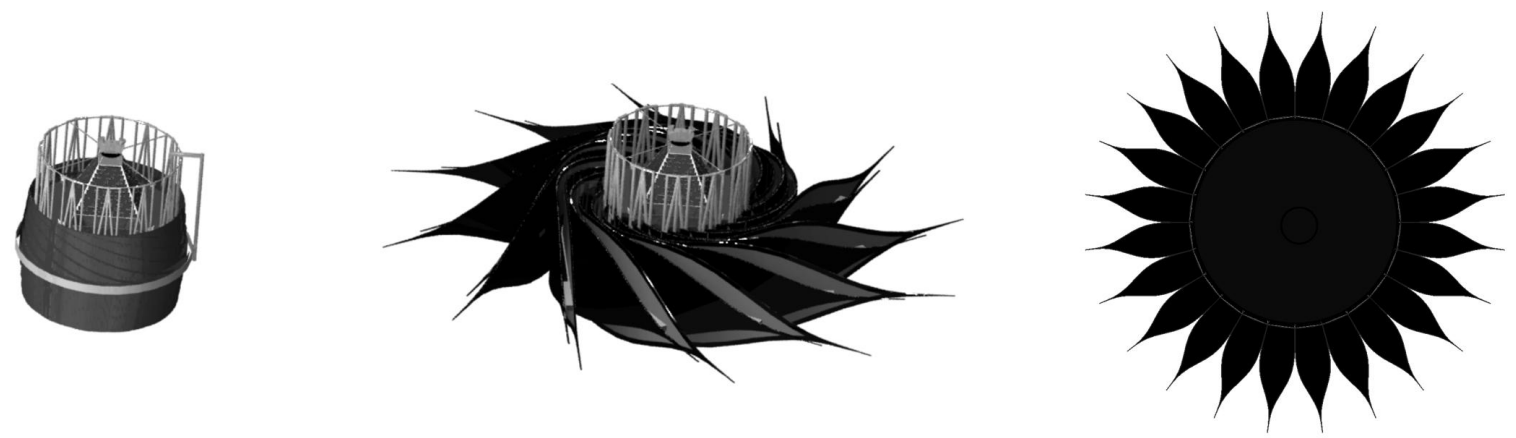

Figure 1. $\mathrm{O}_{3}$ occulter schematic. (a) Stowed configuration. (b) Intermediate deployment step. (c) Occulter profile.

The occulter is designed via optimization ${ }^{11}$ to provide starlight supression at the level of $1 \times 10^{-12}$ at the telescope image plane, with an error budget such that planets can be detected up to 26 magnitudes fainter than their companion stars. Following the methodology developed for THEIA, the occulter is designed to provide this suppression level over a limited bandpass - 250 to $550 \mathrm{~nm}$ (henceforth, the blue band). However, by halving the separation distance between the occulter and telescope, suppression is provided over 500 to $1100 \mathrm{~nm}$ (the red band), at the cost of doubling the IWA. The corresponding telescope - occulter separations are thus $38960 \mathrm{~km}$ for the blue band, and $19480 \mathrm{~km}$ for the red band. The $\mathrm{O}_{3}$ occulter employs a different mechanical design from previous concepts, relying on petals that unfurl and rigidize, unlike the THEIA occulter, which was composed of rigid petals that unfolded from the occulter's central disk (Figure 1). This has the benefit of giving the occulter a much more compact stowed configuration, which allows for the occulter and telescope to be stacked in the fairing of a single launch vehicle, such as the Atlas V or Delta IV. For detailed discussion on the occulter design and development, see Ref. 12.

As with previous free-flying occulter concepts, the telescope and occulter will be placed in orbits about the L2 point to take advantage of the near-constant availability of sunlight and stable space environment. Unlike previous concepts, however, $\mathrm{O}_{3}$ is designed such that the majority of the retargeting work will be done by the telescope spacecraft, rather than the occulter spacecraft. Retargeting is modeled as performed with a pair of arcjet thrusters that have $600 \mathrm{~s}$ specific impulse and a combined thrust of $500 \mathrm{mN}$. The telescope spacecraft is estimated to have a dry mass of $2000 \mathrm{~kg}$, and the occulter spacecraft has a dry mass of approximately 1900 $\mathrm{kg}$. This results in an average value of 8 days to change between the two occulter - telescope separations (at full burn), and an average of 16 days to transfer between targets (when such transfers are limited to a 30 day maximum). These parameters are discussed further in $\S 4.1$.

The telescope optical system is modeled as having two reflections, to a high QE (0.91 peak) science camera with $10 \mu$ pixels. The camera is conservatively assumed to have a read noise of 2 electrons/read, with a dark rate of 0.001 photons/s. Detections are performed in the blue band (300 $\mathrm{nm}$ bandpass), and the contribution due 
to exozodiacal light is held constant at 1.55 local zodis (the historical average of the solar system). In addition to optical attenuation due to telescope optics, some of the planet light may be blocked by the occulter, when the planet's angular distance from the star is less than the IWA, as shown in Figure 2. Because planet light still passes through the gaps between the occulter's petals, it should be possible to image planets inside the geometric IWA. This allows us to consider mission scenarios where imaging is attempted down to 75 mas, where the attenuation due to the occulter is only 0.72 .

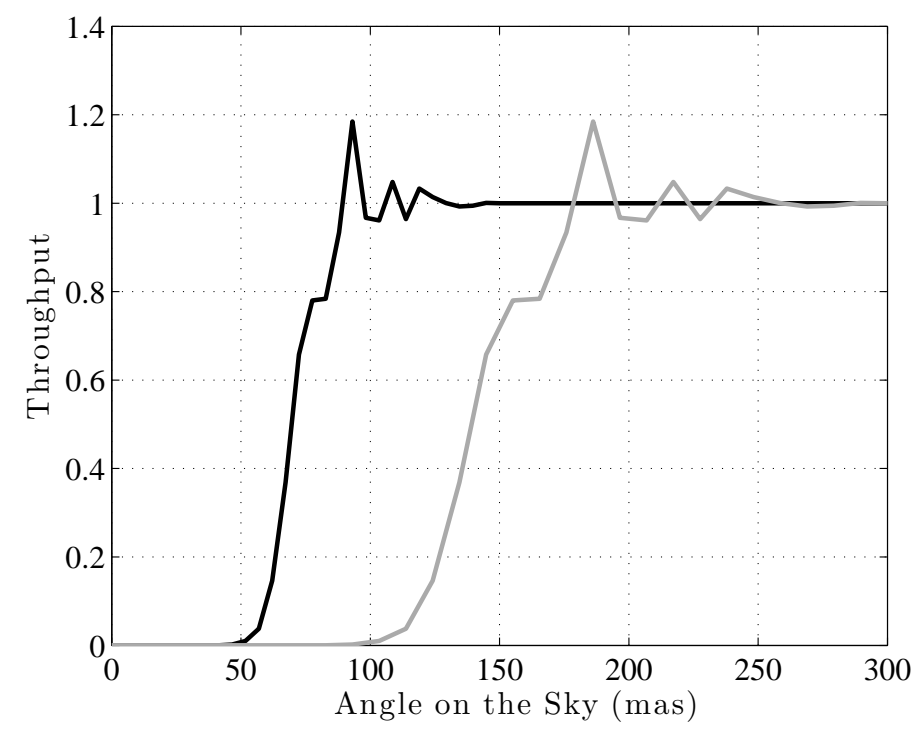

Figure 2. Occulter to telescope throughput as a function of sky angle. The two curves represent the two separations for IWAs of 90 mas (black) and 180 mas (grey). Throughputs greater than unity occur when the occulter concentrates light into the telescope aperture which otherwise would have bypassed the telescope.

\section{SCIENCE CAPABILITIES}

In addition to the basic task of identifying exoplanets, $\mathrm{O}_{3}$ will be able to provide valuable science data with its multi-spectral photometric capability. Each of the two bands made available by the two occulter-telescope separations will be split with four filters, as summarized in Table 1.

Table $1 . \mathrm{O}_{3}$ filters.

\begin{tabular}{ccc}
\hline \hline Filter \# & Red & Bandpass $(\mu)$ \\
& $0.25-0.31$ & $0.5-0.7$ \\
1 & $0.31-0.4$ & $0.7-0.9$ \\
2 & $0.4-0.48$ & $0.9-1.1$ \\
3 & $0.48-0.55$ & Narrowband \\
4 & ${ }^{\dagger}$ The specific narrowband filter selection will be informed by results from transit pho- \\
tometry, which should help pinpoint the most interesting and common giant planet \\
near-IR features, but will most likely coincide with a methane absorption feature.
\end{tabular}

While not providing the same amount of data as spectroscopy, recent studies have shown that multi-band photometry could potentially provide us with much useful information about exoplanets. ${ }^{13,14}$ In the case of the nearest targets $(5-10 \mathrm{pc})$, where time-resolved photometry will be possible, we can infer the rotational periods 
of detected planets ${ }^{14}$ and construct binned light curves, such as the ones simulated in Figure 3 . From these, we can construct color-color plots, which can be used to differentiate between different surface-cover types, as shown in Figure 4. The color differences, defined solely by the magnitude difference between various bands, allow us to infer the presence of oceans and continents from purely photometric observations. ${ }^{13,15}$
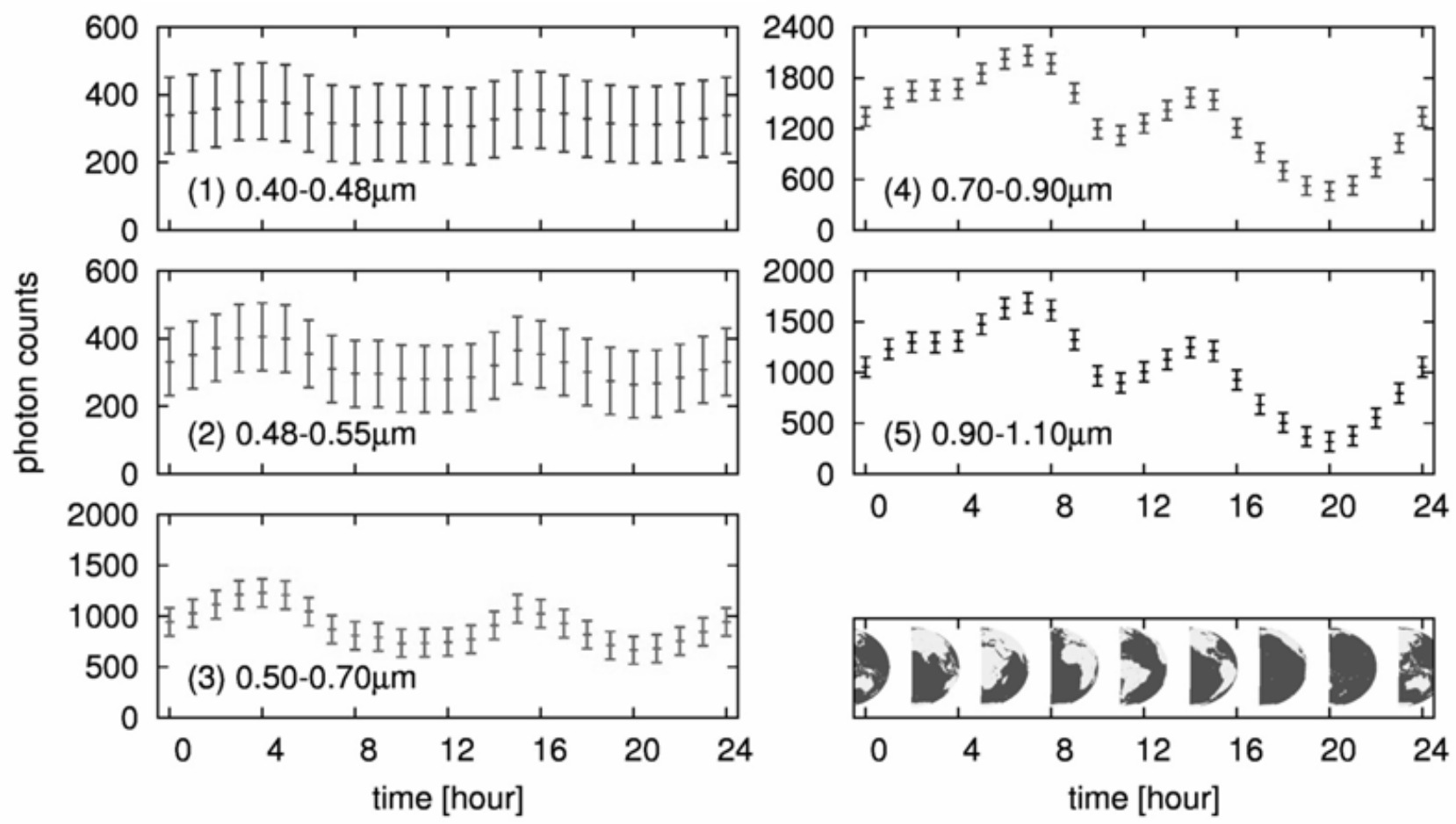

Figure 3. Light curves of a cloudless Earth-twin, using bands Blue 3-4 and Red 1-3 for a two week observation of one hour exposures, folded by the assumed spin rotation period. The bottom right-hand panel shows corresponding views of the surface at each epoch. ${ }^{13}$

Even in cases where only globally averaged photometry will be available, there is much to be learned. Figure 5 (a) shows the apparent albedo spectrum of the Earth as it would be registered by the $\mathrm{O}_{3}$ visible band filters. Although the narrow spectral features are averaged out, the differences between the various filters still allows us to compare colors and identify major color contributors such as Rayleigh scattering. Furthermore, because of the large near-UV atmospheric opacity produced by even small concentrations of ozone, ${ }^{16}$ the first two blue filters give $\mathrm{O}_{3}$ the capability to recognize planets with atmospheric ozone - a probable biomarker. ${ }^{17}$ Figure 5(b) shows a simulation of the relative flux readings that would be recorded by these filters when viewing an Earth-type atmosphere as opposed to a Venus-type atmosphere. The difference in signatures is large, and is difficult to explain by alternative mechanisms.

In addition to information gained from photometric analyses, $\mathrm{O}_{3}$ can also be useful in characterizing planetary orbits. The fidelity of orbital characterizations depends heavily on the number of times a planet is detected, and thus is partially a function of the number of observations that can be made of each target. It is also a function of the precision of the measurement of the star - planet separation, which itself depends on the telescope point spread function (PSF). In both these respects, $\mathrm{O}_{3}$ is aided by it's relatively small occulter, but the problem is made harder by the smaller telescope aperture. The smaller telescope - occulter separation causes retargeting slews to be shorter, on average, but the limited amount of payload capacity limits the fuel mass, thereby shortening mission life if slews are executed with too large a burn. The smaller telescope aperture also means that integration times for both detection and characterization are increased, and the PSF sharpness is lower, making subpixel centroiding less precise. Despite these difficulties, $\mathrm{O}_{3}$ remains a useful tool for constraining planetary orbits and 


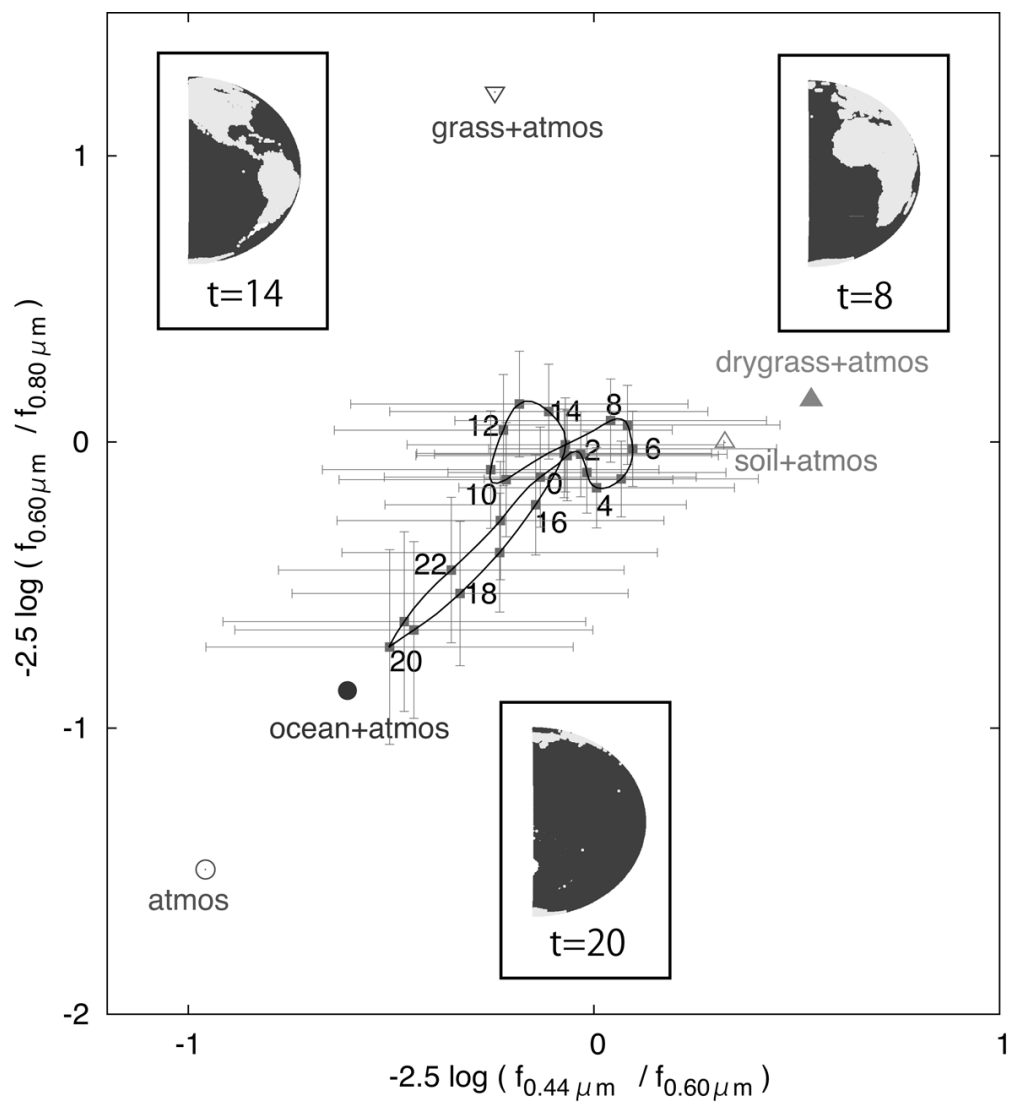

Figure 4. Light curve trajectory from Figure 3 plotted on a color-color diagram using color differences between bands Blue 3 and Red 1 and bands Red 1 and Red 2. The numbers indicate the epoch of observation (corresponding with Figure 3), while the labeled points are typical values for various types of surfaces. ${ }^{13,15}$

helping determine whether detected objects lie within a star's habitable zone (HZ). For further discussion refer to Ref. 20.

While we have not designed a general astrophysics instrument for $\mathrm{O}_{3}$, the basic telescope is highly capable. With a wide field and nearly the same collecting area as the Hubble Space Telescope, $\mathrm{O}_{3}$, equipped with a wide-field camera, should be capable of carrying out a rich program of optical and near-UV science with higher resolution at wavelengths below $7 \mu$ than the James Web Space Telescope (JWST). Furthermore, any planet-finding observatory that relies on an occulter will necessarily have periods of time when the spacecraft is being slewed to acquire a new target, thereby guaranteeing dedicated instrument time for general astrophysics observations. In the case of $\mathrm{O}_{3}$, the simulations discussed in $\S 4$ imply that at least $50 \%$ of the mission time will be available for non-exoplanet science.

\section{MISSION SIMULATIONS}

\subsection{Mission Scenarios and Configurations}

The basic observatory design described in $\S 2$ can be deployed and operated in multiple different ways. These include differing sets of mission rules, operating parameters, and scheduling choices. One method for simulating and evaluating missions is to perform a Monte Carlo analysis by generating ensembles of simulated mission timelines (sometimes referred to as Design Reference Missions, or DRMs) and gathering statistics on metrics such as the number of planets detected or photometrically characterized. This approach is extensively described in Ref. 21.

In these simulations, it is also possible to test the effects of varying parameters. Some of these include: 


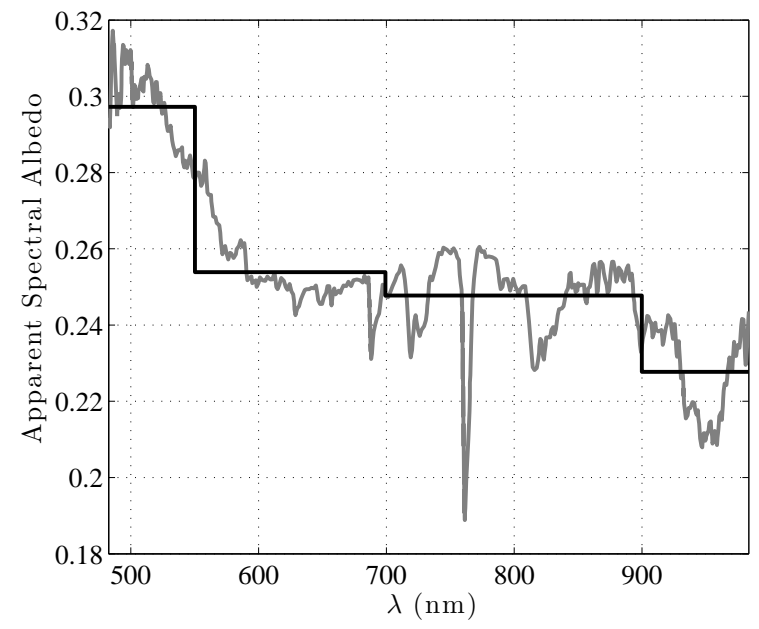

(a)

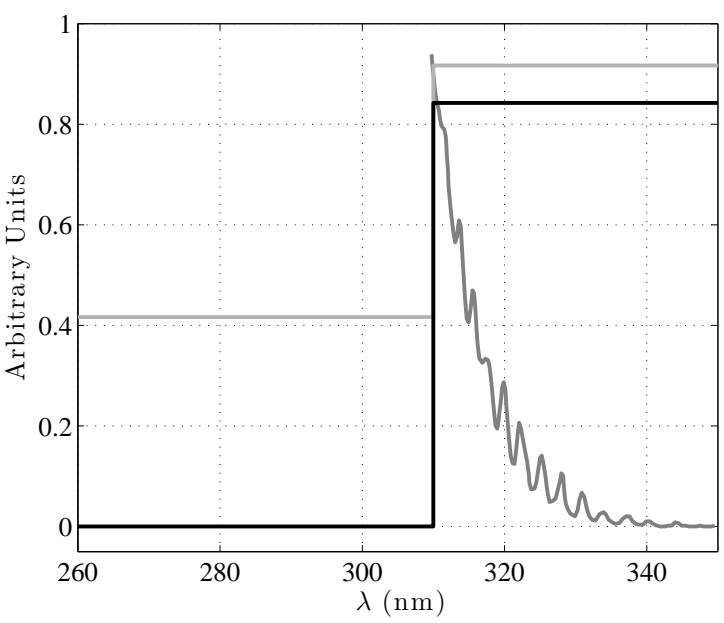

(b)

Figure 5. (a) Apparent albedo spectrum of the Earth, ${ }^{18}$ overlaid with averaged spectrum corresponding to $\mathrm{O}_{3}$ bands. (b) Relative globally averaged fluxes as seen by $\mathrm{O}_{3}$ filters Blue 1 and 2 when observing an Earth-twin (black) and a Venus-twin (grey). The dark-grey curve represents the ozone opacity as a function of wavelength in Earth's atmosphere for a total columnar ozone thickness of 370 matm-cm. ${ }^{16,19}$ These plots show that while specific spectral features like the highly pronounced $\mathrm{O}_{2}$ line at $760 \mathrm{~nm}$ cannot be detected, we can make inferences about atmospheric composition and ground cover by comparing the ratios of different bands. In particular, the small amount of ozone in Earth's atmosphere makes the flux in the Blue 1 band nearly zero, greatly differentiating it from the photometric signal of Venus.

- False Alarm probability and Missed Detection rate - these, along with the instrument's maximum contrast, determine the amount of time spent observing each system before determining that no observable planet is in the field of view at that time. These are set to produce zero false alarms over the mission lifetime, and to allow an average of 1 missed detection per 1000 observations.

- Integration cutoff - this determines the maximum amount of time that can be spent observing any target. While certain faint planets can be detected, achieving sufficient photometric signal to noise may take too much time. Since high-latitude targets are observable for up to 6 months at a time, it is possible that attempting to photometrically characterize one planet may come at the cost of detecting multiple others.

- Maximum slew time - to help determine which target should be observed next, it is useful to limit the amount of time that should be spent in transit. This not only increases the portion of the mission which is spent on observations, but also means that lower probability targets get visited more often, which raises the overall probability of detections in simulations where few stars have Earth-like planets.

- Default burn portion - in addition to limiting the amount of time spent on slewing between targets, we can also define the default portion of the maneuver that is used for accelerating and decelerating (with the remainder used for coasting). This allows the algorithm responsible for selecting the next target to estimate slew times, and then adjust the burn portion as necessary. This parameter also helps save fuel, as fuel use increases roughly as the square of the acceleration time.

- Target selection cost function weights - these determine the emphasis that is placed on factors determining the next target to be observed. Currently, six factors are considered: the single visit completeness of the target ${ }^{22}$ the transit distance that must be covered to properly position the spacecraft, whether a target has been previously observed, whether a target has been scheduled for another observation in the current epoch, whether a target is close to entering or exiting a keepout zone, and the number of previous detections at that target. The weighting is determined for each specific mission architecture by running a feedback loop 
to maximize the sum of the total number of detections, number of unique detections (individual planets) and number of photometric characterizations.

We considered 5 year missions (simulations were stopped at 5 years, or whenever the fuel ran out), with photometric characterization to an SNR of 3. Detection integration times were determined by assuming that the faintest detectable planets would be 26 magnitudes below their parent star, and planets would be searched for down to angular separations of 90 mas or 75 mas. Mission rules were set either to attempt photometric characterization at both separations, or only in the blue band. Characterization was required for all newly discovered planets, but, for cases where both separations were used, the red band could be characterized in a different observation from the blue. Finally, multiple simulations were run with varying default burn portions and maximum slew times to locally optimize these parameters.

\subsection{Single-Planet Systems}

Following Ref. 21, the first batch of simulations considered only single planet systems of Earth-twins in the habitable zone, with a varying expected occurrence rate $\left(\eta_{\oplus}\right)$. While this does not constitute a good model of the universe, it is much less computationally expensive than the multiple planet case, and is useful in constraining and optimizing the parameters listed in $\S 4.1$. These results can then be applied to simulations with more realistic planetary distributions.

A major component of the simulation is the selection of a target pool. This is done by finding all of the targets about which the observatory could detect an Earth-like planet, and then filtering that list so that the targets have some minimum single visit completeness. Fewer total targets generally increase the total number of detections, while reducing the number of unique detections. They also increase fuel use for slews, since targets are further apart, on average, and longer burns must be used to keep the mission time below the selected five year maximum. In simulations where mission rules only call for photometric characterization in the case of newly discovered planets, the amount of fuel used for stationkeeping is decreased due to the lower number of unique detections. Finally, in cases where $\eta_{\oplus}$ is low, the probability that no planets at all will be detected is increased by decreasing the target pool. These effects are shown for one of the $\mathrm{O}_{3}$ configurations in Figure 6 . Automatically generated target pools (based on minimum completeness) smaller than the ones shown here tend to perform worse in terms of both total and unique detections. However, it is possible to improve performance by considering not only the single visit completeness of the target systems, but also their placement. Adding strategically located systems with slightly lower completeness values can actually increase the overall expected number of detections as it leads to smaller average transition slews and a larger number of overall observations. Because the target selection algorithm used here has not yet been updated to automatically take this strategy into account, the final target pools used were manually edited to include up to 11 targets below the minimum completeness cutoff.

We next compare mission simulations where photometry is done in only the blue band, or in both bands, and where planet observations are either made only outside the geometric IWA, or down to 75 mas. Figure 7 shows that, as expected, missions which attempt photometry in both bands will have fewer detections than mission characterizing only in the blue, due to the time required to change the telescope-occulter distance. Furthermore, missions which attempt to characterize planets through the occulter's petals do much better in getting red band photometry as the doubled inner working angle at 90 mas makes most HZ planets unobservable.

As seen from Figure 8, increasing the default burn portion has the predictable effect of decreasing the number of total detections, while greatly shortening the mission duration. While this would ordinarily be a reason to maintain a minimum default burn portion, a shorter mission lifetime with almost the same science return may actually be quite attractive when discussing smaller class missions. Both operating costs and the probability of single event failure decrease with the mission span.

\subsection{Multiple-Planet Systems}

We can expand our simulations, and improve our confidence in them, by incorporating multiple planet systems. Unfortunately, generating likely exosystems that could also include Earth-like planets is fairly difficult based on our current state of knowledge. While this is an active area of research, ${ }^{20}$ and our knowledge of multi-planet 

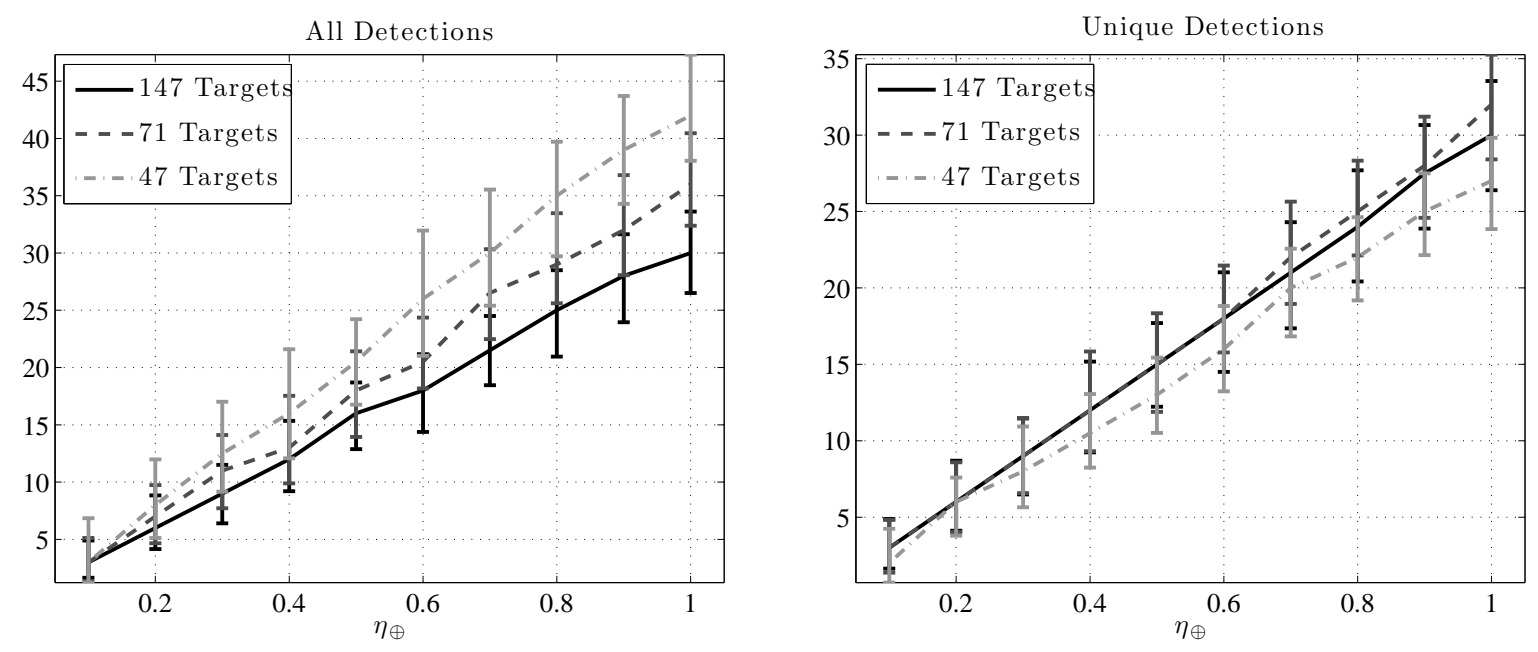

Figure 6. Simulation results for different target pool sizes using single-planet (Earth-twin in the habitable zone) systems. The three values correspond to minimum single visit completeness values of $0.1,0.2$ and 0.3 . Error-bars represent the one-sided deviations of each distribution. Mission rules for these simulations called for photometric characterization of newly discovered planets in the Blue band only.

exosystems is constantly increasing, ${ }^{23}$ we can consider a simpler, intermediate step - exosystems consisting of subsets of the planets in our own solar system. By taking the ephemerides of some of the solar system planets, scaling them so that the planets receive the equivalent flux from the selected target star, rotating them arbitrarily about the assumed line of sight, and removing any residual acceleration of the system barycenter, we were able to generate large numbers of stable multi-planet systems. The frequency of selection of each solar system planet for inclusion was determined by our current knowledge of exoplanet distributions. ${ }^{1,24}$ The implementation of multi-planet systems also required an extension of the decision modeling used in the single-planet case. When observations of a target system produced strong signals which could not be due to a planet in the HZ (i.e., the apparent separation of the discovered object was greater than the outer edge of the target star's HZ), integration was continued until the pre-calculated maximum for an Earth-twin was reached, ${ }^{21}$ or a second source was discovered. Similarly, detections representing planets which could not be in the HZ were not used to calculate re-visit times, and objects whose apparent separation could place them in the HZ, but which were much brighter (lower $\Delta \mathrm{mag}$ ) than an Earth-twin should be were placed on a secondary, lower priority re-visit list.

As an initial test to ensure that this expanded capability was functioning properly, we compared the raw number of detections of each solar system planet analogue, normalized by the frequency with which they were simulated. This is essentially the same as counting the total number of detections of each planet type in simulations where all of the analogues are selected with equal frequencies, and gives a sense of the relative detectability of the various planets in our solar system. Figure 9(a) shows a histogram of these results, using the 90 mas IWA, 2 band photometry configuration. As expected, Jupiter and Saturn-like planets, due to their size, relatively high albedo and placement, will be detected much more frequently than any other planet type. Earths will be detected more frequently than Venus or Mars analogues due to their size and placement, and ice giants like Neptune will be difficult to detect unless they orbit much closer to their stars than Neptune does to the sun. This histogram also implies that if Earth-twins and Jupiter-twins occur at the same rate in our universe, an observatory like $\mathrm{O}_{3}$ should detect roughly four times as many Jupiters as Earths. We can also use this data set to estimate the probability that a habitable zone candidate (i.e., a detection with an apparent separation less than the outer edge of the target star's HZ) actually is a habitable zone planet. We find that if every exosystem was a scaled copy of the solar system, HZ candidates would represent about $22 \%$ of all detections, and would actually be Earth analogues $43 \%$ of the time. They would be rocky planet analogues (Venus, Earth, or Mars) $83 \%$ of the time, and giant planet analogues (Jupiter, Saturn, or Uranus) $17 \%$ of the time.

While basing our simulated exosystems on subsets of solar system planets does not ensure that we are correctly 

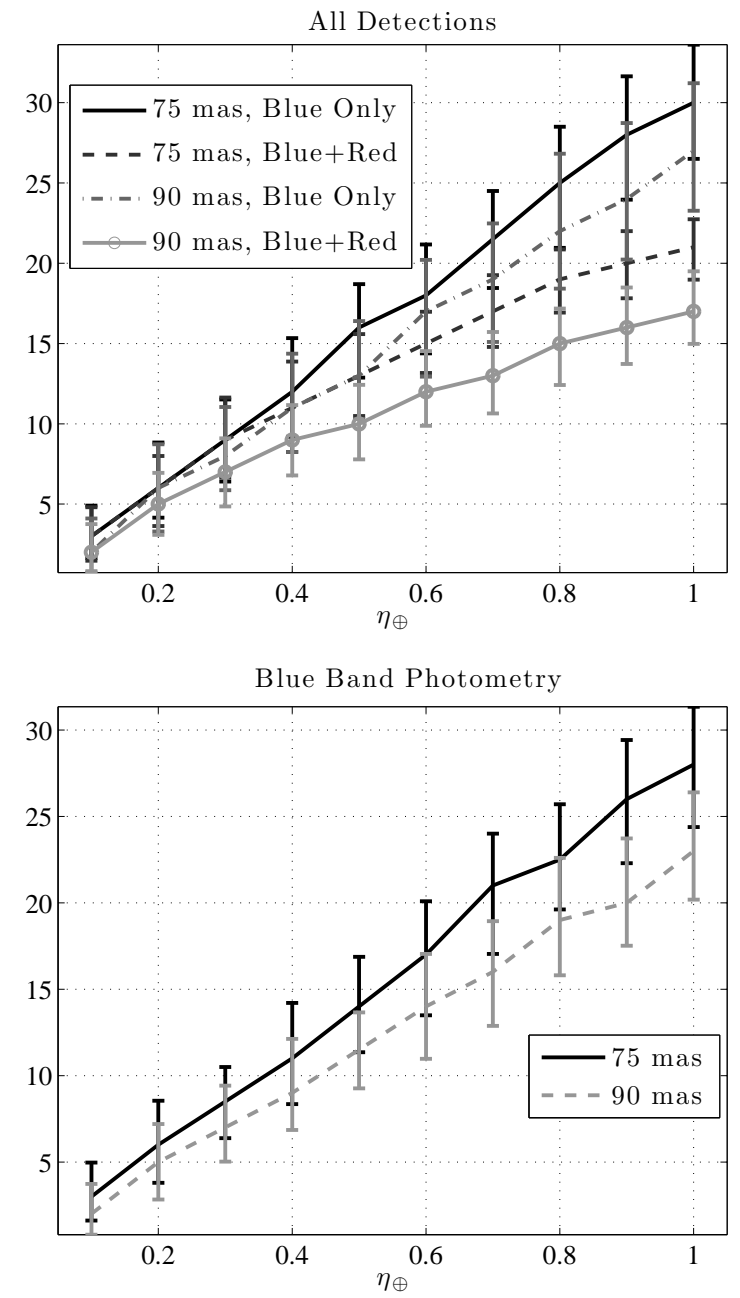
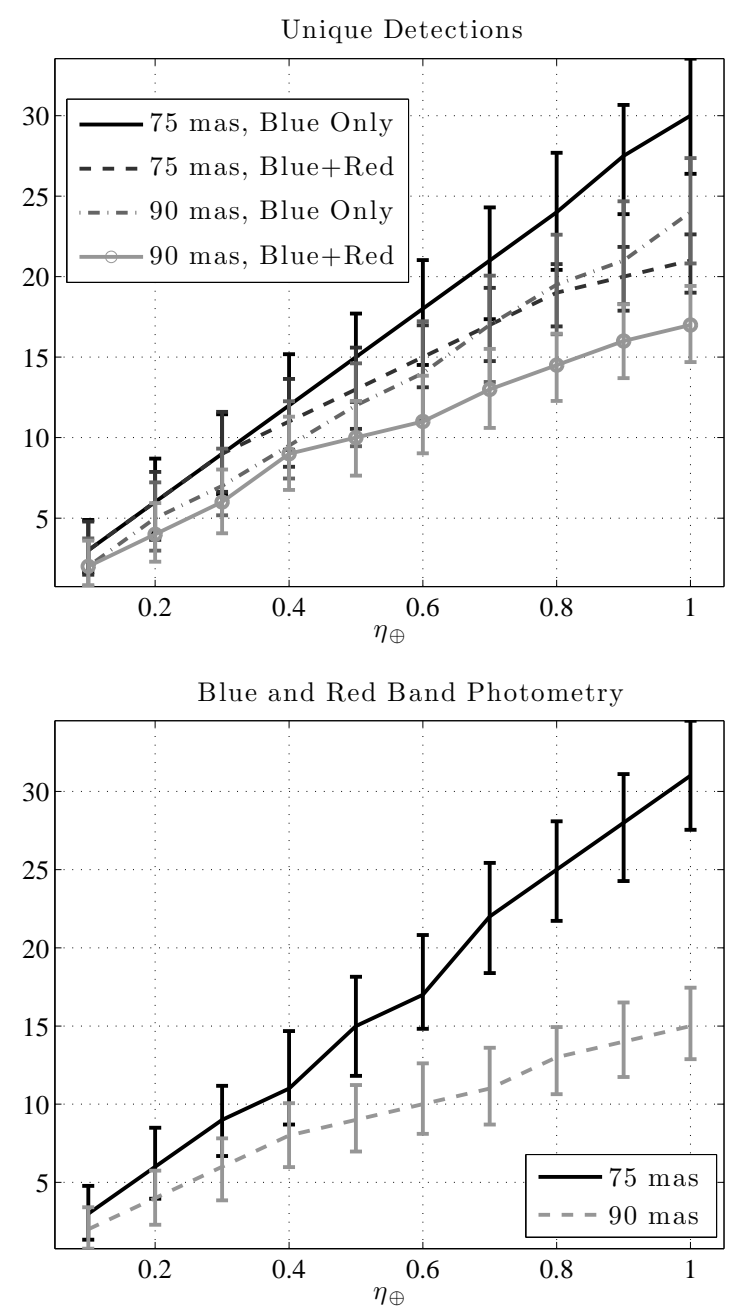

Figure 7. Simulation results for $\mathrm{O}_{3}$ performing either Blue or Red + Blue band photometry, and searching for planets down to angular separations of 90 or 75 mas in universes with single-planet systems (Earth-twins in the habitable zone). The top left plot compares the total numbers of detections, the top right plot compares the unique numbers of detections, the bottom left compares the number of photometric characterizations (to SNR of 3) for configurations doing Blue band photometry only, and the bottom right compares the number of complete photometric characterizations for configurations doing both Blue and Red band photometry. The much larger difference in the two curves in the final two plots is due to the extreme difficulty of getting complete photometry in the Red band where the geometric IWA is 180 mas for the 90 mas case.

modeling the true distribution of planets in our universe, it does allow us to consider a very important question that cannot be addressed with the single-planet system model. Namely, in cases where a repeat observation of a target system where a planet has been previously discovered yields another detection, how can we determine whether we are seeing the same planet as before, or a new one? This is extremely important for confirming that initial detections really represent bodies that belong to the target star system, and to ensure that the data points we use for orbit fitting actually belong to the same data set.

One way to answer this question is to apply simple Bayesian theory. The probability that a current observation is of the same planet as a previous one is given by

$$
p\left(\mathbf{p}_{k} \mid \mathbf{p}_{k-1},\left\{\mathbf{p}_{k}, \mathbf{p}_{k-1}\right\} \in \gamma_{k-1}\right)=\frac{p\left(\mathbf{p}_{k-1},\left\{\mathbf{p}_{k}, \mathbf{p}_{k-1}\right\} \in \gamma_{k-1} \mid \mathbf{p}_{k}\right) p\left(\mathbf{p}_{k}\right)}{p\left(\mathbf{p}_{k-1}\right)}
$$



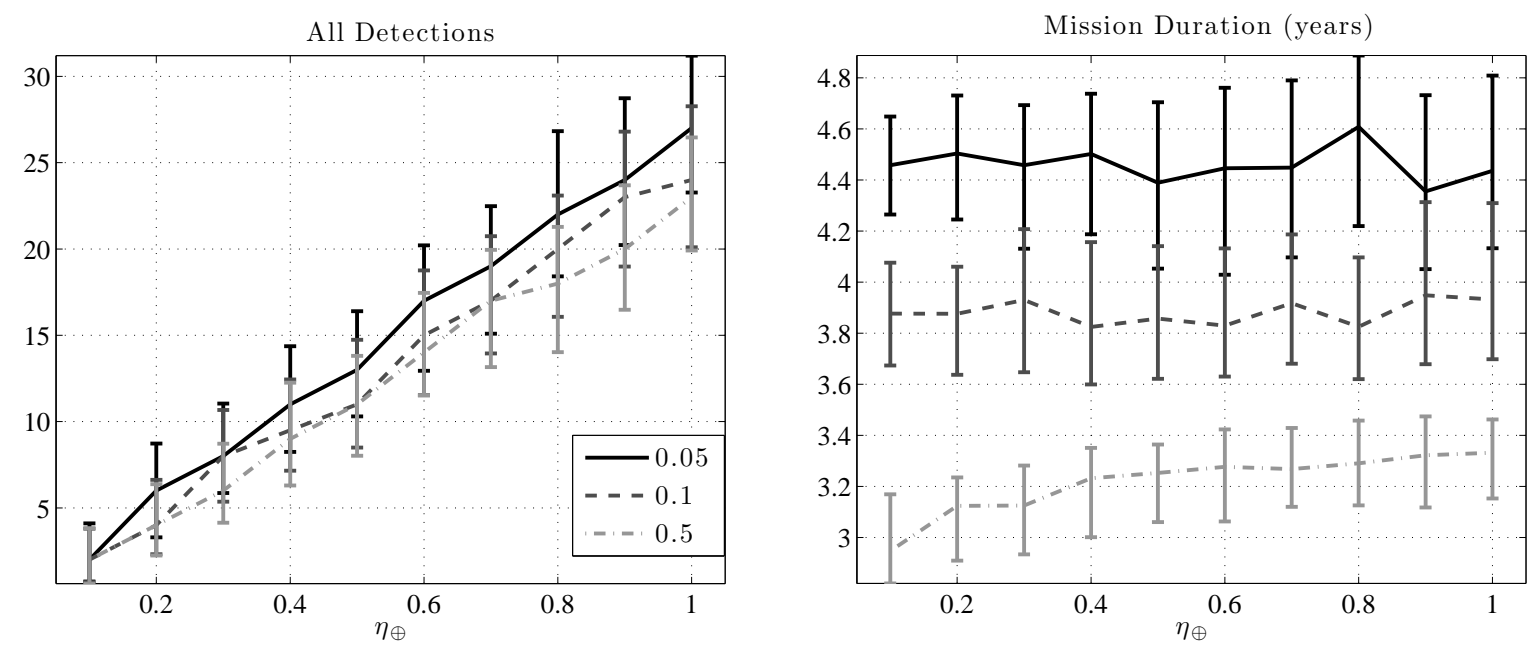

Figure 8. Simulation results for different default burn portions using the 2 band photometry, 75 mas IWA configuration.

where $\mathbf{p}_{i}$ is the parameter set recorded in the $i$ th observation (i.e., star-planet separation and difference in magnitude), and $\gamma_{i}$ is the space of all possible parameter sets belonging to a single planet that includes $\mathbf{p}_{i}$. The likelihood, $p\left(\mathbf{p}_{k-1},\left\{\mathbf{p}_{k}, \mathbf{p}_{k-1}\right\} \in \gamma_{k-1} \mid \mathbf{p}_{k}\right)$, is approximately equivalent to the goodness-of-fit of a stable orbit to both data points, while the priors, $p\left(\mathbf{p}_{k}\right)$ and $p\left(\mathbf{p}_{k-1}\right)$, can be calculated from the completeness distribution of the target star. ${ }^{21}$

To apply this idea, we had the simulation algorithm classify detections made during repeat observations of targets where planets had previously been detected as newly discovered planets whenever the probability of a repeat detection was less than $50 \%$. Figure 9 (b) shows the average number of each planet analogue classified as unique in simulation over 100 simulations using a 44 star target pool with 90 mas IWA and 2 band photometry required for each planet classified as a new detection. The algorithm correctly identified planets as new detections in approximately $60 \%$ of applicable cases (detections during repeat observations of targets with prior detections). This led to the apparent discovery (according to the algorithm's internal bookkeeping) of approximately $45 \%$ of included Earth-like planets, whereas earlier single-planet system studies indicate that this value should be less than $33 \%$. At the same time, according to the algorithm's classification, it discovered slightly more Jupiters, on average, than were included in the simulation, while in reality the true average rate of discovery was closer to 85\%. Because of the limited target pool size, each star in the pool was observed at least once in most of the simulation instances, and the high visibility of Jupiter analogues made them easy to detect.

Despite the coarseness of these early results, this is a useful first step in bounding this problem, and provides an initial formalism for its treatment. At the same time, these results show the viability of the $\mathrm{O}_{3}$ design. We see that despite the increased difficulty of tracking multiple planets, and extra time spent characterizing the frequently found giant planets, $\mathrm{O}_{3}$ is still likely to find one or more Earth-like planets, and several times that many giant planets. We see that the single-planet system simulation results, while optimistic, are of the same order of magnitude as the multiple-planet system results, allowing us to continue to use the simpler simulations to quickly compare a wider variety of different mission configurations before turning to more computationally intensive models.

\section{CONCLUSIONS}

We have demonstrated that it is possible to design a direct-detection exoplanet observatory on a scale significantly below the resource level of a flagship class mission. Our particular concept, $\mathrm{O}_{3}$, is capable not only of detecting planets down to Earth-size, but also returning valuable science data about them. We consider this design, or something similar, a viable alternative to previously proposed larger-scale missions, and a reasonable allocation of resources in the pursuit of expanded exoplanet science. Our simulations suggest that we can expect to find on 


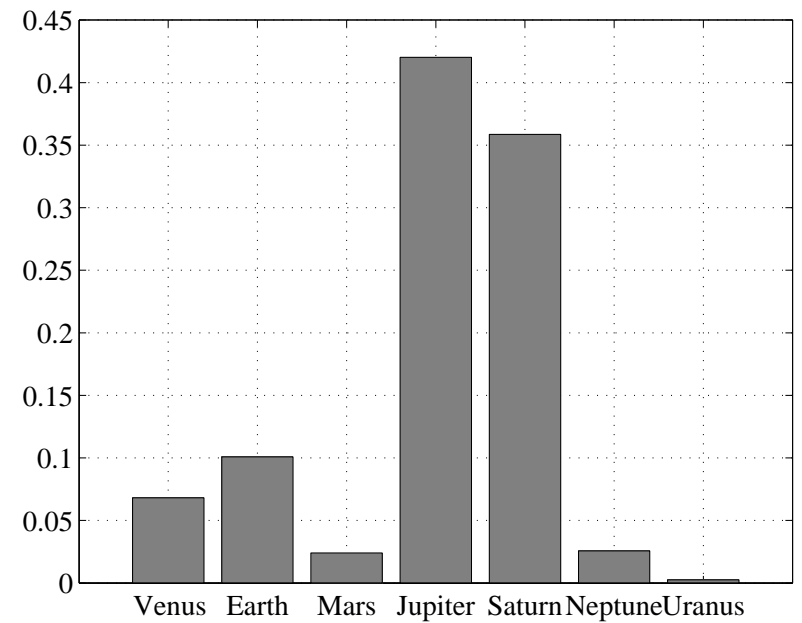

(a)

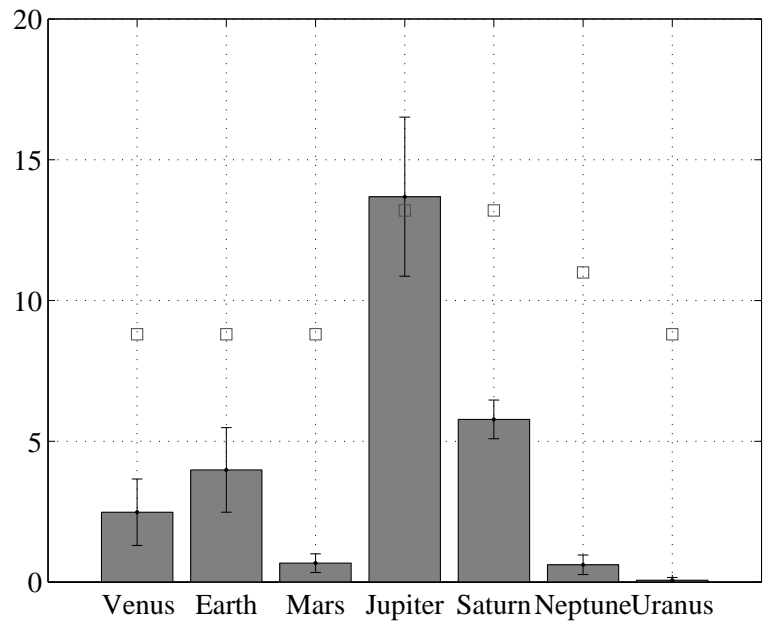

(b)

Figure 9. Results for 100 mission simulations using the 2 band photometry, 90 mas IWA configuration. (a) Total number of detections of each solar system planet analogue, normalized by the frequency with which they were simulated. This is used primarily as a test to ensure the multi-planet simulation capability is working as expected, but can also be useful in determining the relative detectability of the analogues - i.e., if Jupiters and Earths are distributed with equal frequency, $\mathrm{O}_{3}$ should detect 4 times as many Jupiters as Earths, on average. (b) Average number of unique analogues found. Errorbars represent the standard deviation of the distribution. The squares indicate the average number of each analogue type that was included in the simulations.

the order of 5 Earth-twins if $\eta_{\oplus}$ if 0.3 , and photometrically characterize a majority of them in both the red and blue bands to an SNR of 3. At the same time, even in configurations with the largest total amount of integration time devoted to exoplanet science, over $50 \%$ of the mission time will still be available for general astrophysics.

We have also expanded on previously existing exoplanet mission analysis techniques, thereby improving their fidelity and the believability of produced results, particularly in the realm of multi-planet systems. While the question of how to determine whether a detection is of the same object as a previous one, or of a different planet belonging to the same system, is still an open one, the method outlined in $\S 4.3$ provides a starting point which hopefully can be refined in future studies.

\section{REFERENCES}

[1] Cumming, A., Butler, R. P., Marcy, G. W., Vogt, S. S., Wright, J. T., and Fischer, D. A., "The Keck planet search: detectability and the minimum mass and orbital period distribution of extrasolar planets," PASP 120, 531-554 (2008).

[2] Borucki, W. J., Koch, D., Basri, G., Batalha, N. M., Brown, T., Caldwell, D. A., Caldwell, J., ChristensenDalsgaard, J., Cochran, W., DeVore, E., Dunham, E., Dupree, A., Gautier, T., Geary, J., Gilliland, R., Gould, A., Howell, S., Jenkins, J., Kjeldsen, H., Kondo, Y., Latham, D., Lissauer, J., Marcy, G., Meibom, S., Monet, D., Morrison, D., Sasselov, D., and Tarter, J., "Kepler Planet Detection Mission: Introduction and First Results," in [American Astronomical Society Meeting Abstracts], American Astronomical Society Meeting Abstracts 215, 101.01 (Jan. 2010).

[3] Marois, C., Macintosh, B., Barman, T., Zuckerman, B., Song, I., Patience, J., Lafreniere, D., and Doyon, R., "Direct imaging of multiple planets orbiting the star hr 8799," Science 322(5906), 1348 (2008).

[4] Kalas, P., Graham, J., Chiang, E., Fitzgerald, M., Clampin, M., Kite, E., Stapelfeldt, K., Marois, C., and Krist, J., "Optical images of an exosolar planet 25 light-years from earth," Science 322(5906), 1345 (2008).

[5] Traub, W., Levine, M., Shaklan, S., Kasting, J., Angel, J., Brown, M., Brown, R., Burrows, C., Clampin, M., Dressler, A., et al., "TPF-C: status and recent progress," in [Proceedings of SPIE], 6268, 62680T (2006). 
[6] Beichman, C., Lawson, P., Lay, O., Ahmed, A., Unwin, S., and Johnston, K., "Status of the terrestrial planet finder interferometer (tpf-i)," in [Proceedings of SPIE], 6268, 62680S (2006).

[7] Spergel, D., Kasdin, J., Belikov, R., Atcheson, P., Beasley, M., Calzetti, D., Cameron, B., Copi, C., Desch, S., Dressler, A., Ebbets, D., Egerman, R., Fullerton, A., Gallagher, J., Green, J., Guyon, O., Heap, S., Jansen, R., Jenkins, E., Kasting, J., Keski-Kuha, R., Kuchner, M., Lee, R., Lindler, D., Linfield, R., Lisman, D., Lyon, R., Malhotra, S., Mathews, G., McCaughrean, M., Mentzel, J., Mountain, M., NIkzad, S., O'Connell, R., Oey, S., Padgett, D., Parvin, B., Procashka, J., Reeve, W., Reid, I. N., Rhoads, J., Roberge, A., Saif, B., Scowen, P., Seager, S., Seigmund, O., Sembach, K., Shaklan, S., Shull, M., and Soummer, R., "THEIA: Telescope for habitable exoplanets and interstellar/intergalactic astronomy," in [AAS Meeting 213], 458(04), American Astronomical Society (2009).

[8] Cash, W., Kasdin, J., Seager, S., and Arenberg, J., "Direct studies of exo-planets with the New Worlds Observer," in [Proceedings of SPIE], 5899, 58990S (2005).

[9] Kasdin, N., Spergel, D., Vanderbei, R., Cady, E., Savransky, D., Lisman, D., Shaklan, S., Lee, R., Egerman, R., Matthews, G., et al., "A medium size mission for finding and characterizing terrestrial exoplanets with an external occulter and a conventional space telescope," in [American Astronomical Society Meeting Abstracts], 215 (2010).

[10] Shaklan, S. B., Noecker, M. C., Lo, A. S., Glassman, T., Dumont, P. J., Jordan, E. O., Kasdin, N. J., Webster C. Cash, J., Cady, E. J., and Lawson, P. R., "Error budgeting and tolerancing of starshades for exoplanet detection," in [Proceedings of SPIE], 7731 (2010).

[11] Vanderbei, R. J., Cady, E., and Kasdin, N. J., "Optimal Occulter Design for Finding Extrasolar Planets," The Astrophysical Journal 665(1), 794-798 (2007).

[12] Thomson, M. W. and Lisman, P. D., "Occulting ozone observatory starshade design and development," in [Proceedings of SPIE], $\mathbf{7 7 3 1}$ (2010).

[13] Fujii, Y., Kawahara, H., Suto, Y., Taruya, A., Fukuda, S., Nakajima, T., and Turner, E. L., "Colors of a Second Earth: Estimating the Fractional Areas of Ocean, Land, and Vegetation of Earth-like Exoplanets," The Astrophysical Journal 715, 866-880 (June 2010).

[14] Palle, E., E., Ford, E. B., Seager, S., MontAnes-Rodri'guez, P., and Vazquez, M., "Identifying the rotation rate and the presence of dynamic weather on extrasolar earth-like planets from photometric observations," The Astrophysical journal 676(2), 1319-1329 (2008).

[15] Kawahara, H. and Fujii, Y., "Global mapping of earth-like exoplanets from scattered light curves," Arxiv preprint arXiv:1004.5152 (2010).

[16] Schachter, J., "Ozone absorption bands in the $3100 \AA$ - $3400 \AA$ region," Publications of the Astronomical Society of the Pacific 103, 457-460 (1991).

[17] Heap, S., "Detecting Biomarkers in ExoPlanetary Atmospheres with Terrestrial Planet Finder," in [EAS Publications Series], 41, 517-520 (2010).

[18] Montanes-Rodriguez, P., "Globally integrated measurements of the earth's visible spectral albedo," Astrophysical journal 629(2), 1175 (2005).

[19] Anderson, R., Pipes, J., Broadfoot, A., and Wallace, L., "Spectra of Venus and Jupiter from 1800 to 3200 A," Journal of Atmospheric Sciences 26, 874-888 (1969).

[20] Pravdo, S. H., Shaklan, S. B., and Lisman, P. D., "Orbiting starshade's performance in observations of potential earth-like planets," in [Proceedings of SPIE], 7731 (2010).

[21] Savransky, D., Kasdin, N. J., and Cady, E., "Analyzing the designs of planet finding missions," PASP 122, 401-419 (April 2010).

[22] Brown, R. A., "Single-visit photometric and obscurational completeness," ApJ 624, 1010-1024 (2005).

[23] Steffen, J. H., Batalha, N. M., Borucki, W. J., Buchhave, L. A., Caldwell, D. A., Cochran, W. D., Endl, M., Fabrycky, D. C., Fressin, F., Ford, E. B., Fortney, J. J., Haas, M. J., Holman, M. J., Isaacson, H., Jenkins, J. M., Koch, D., Latham, D. W., Lissauer, J. J., Moorhead, A. V., Morehead, R. C., Marcy, G., MacQueen, P. J., Quinn, S. N., Ragozzine, D., Rowe, J. F., Sasselov, D. D., Seager, S., Torres, G., and Welsh, W. F., "Five Kepler target stars that show multiple transiting exoplanet candidates," ArXiv e-prints (June 2010).

[24] Gould, A., Dong, S., Gaudi, B., Udalski, A., Bond, I., Greenhill, J., Street, R., Dominik, M., Sumi, T., Szymanski, M., et al., "Frequency of solar-like systems and of ice and gas giants beyond the snow line from high-magnification microlensing events in 2005-2008," (2010). 